\title{
SOBRE LA INTEGRAL DE CAMINO EN EL ESPACIO DE FASE DE UNA PARTÍCULA EN UN CAMPO MAGNÉTICO CONSTANTE
}

\author{
Adolfo Villamizar Villamizar, Ariel Becerra Becerra.
}

Grupo Integrar.Facultad de Ciencias Basicas.Departamento de Fisica.Universidad de Pamplona, Colombia

\section{Resumen}

El movimiento de una partícula cargada en un campo magnético constante es analizado por medio de la integral de camino en del espacio fase. Para encontrar el propagador, la acción es expresada en términos del espacio fase y el campo magnético es expresado como el rotacional de un vector potencial que satisface la condición gauge de Coulomb para un campo magnético. Se obtiene la expresión del oscilador armónico unidimensional como expresión matemática que describe el comportamiento de la partícula cargada en el campo magnético constante. Se muestra que el vector potencial tiene un rol fundamental en la simplificación de este resultado.

Palabras clave: integral de camino, partícula en campo magnético, propagador, espacio de fase.

\section{ABOUT THE PHASE-SPACE PATH INTEGRAL OF A PARTICLE IN A CONSTANT MAGNETIC FIELD}

\begin{abstract}
A charged particle moving in a constant magnetic field is analyzed through the space phase path integral. To find the propagator of the particle, the action is expressed in terms of phase space and the magnetic field is represented by a vector potential satisfying the Coulomb gauge condition for a constant magnetic field. The math expression of a onedimensional harmonic oscillator as an expression that describes the behavior of a charged particle in a constant magnetic field is obtained. It is shown that the potential vector play a crucial role in simplifying this problem.
\end{abstract}

Key words: Path integral, propagator, particle in magnetic field, phase space. 
*Para citar este artículo: Villamizar Villamizar A,Becerra Becerra A.Sobre la integral de camino en el espacio de fase de una particula en un campo magnético constante.Revista Bistua. 2016.14(2):36-42

+ Autor para el envió de correspondencia y la solicitud de las separatas: Villamizar Villamizar A.Departamento de Fisica .Universidad de Pamplona,Pamplona, Norte de Santander, Colombia e-mail: a2villamizar@gmail.com

Recibido: Septiembre 052015

Aceptado: Marzo 302016

\section{INTRODUCCIÓN}

Para evaluar la integral de caminos de una partícula de carga $e$ en un campo magnético constante se parte de su comportamiento como un sistema armónico y se emplea en su solución el propagador para un oscilador armónico. Un segundo aspecto a tener en cuenta en el desarrollo de la integral de camino es definir la acción mediante el hamiltoniano, en función de los momentos y las posiciones, en el denominado espacio fase [1], [2], [3]. El campo magnético $\boldsymbol{B}$ se expresa como el rotacional de una función vectorial $\boldsymbol{A}(\boldsymbol{x})$, denominada el vector potencial, que puede ser elegido de acuerdo a las condiciones del problema, sin embargo se emplea la función $\boldsymbol{A}(\boldsymbol{x})=(0, B x, 0)$ que resulta ser la más apropiada, con el campo apuntando en dirección del eje $z$. Este artículo sigue los procedimientos descritos en Path Integrals in Quantum Mechanics, Statistics, Polynter Physics, and Financial Markets de Hagen Kleinert [4], donde se puede encontrar amplia información sobre el tema.

\section{CALCULO DEL PROPAGADOR CON EL VECTOR POTENCIAL $\boldsymbol{A}^{\prime}$}

Seleccionemos el sistema de coordenadas tal que $\boldsymbol{B}=\boldsymbol{\nabla} \times \boldsymbol{A}^{\prime}$, apunte en la dirección del eje $z$, de tal manera que el campo se describe por el vector potencial que tiene las componentes

$$
A_{x}^{\prime}=-\frac{1}{2} B y, \quad A^{\prime}{ }_{y}=\frac{1}{2} B x, \quad A^{\prime}{ }_{z}=0
$$

En general, la acción en el espacio fase se expresa como [5] 
38

$$
s[\boldsymbol{p}, \boldsymbol{x}]=\int_{\boldsymbol{t}_{\boldsymbol{a}}}^{\boldsymbol{t}_{\boldsymbol{b}}} d \tau\left[\boldsymbol{p} \dot{\boldsymbol{x}}-\frac{1}{2 m}\left(\boldsymbol{p}-\frac{\boldsymbol{e}}{\boldsymbol{c}} \boldsymbol{A}\right)^{2}\right],
$$

y el propagador está dado por

$$
\begin{aligned}
\left\langle\boldsymbol{x}_{\boldsymbol{b}}, t_{b} \mid \boldsymbol{x}_{\boldsymbol{a}}, t_{a}\right\rangle & =\int_{-\infty}^{+\infty} \mathcal{D} \boldsymbol{x}(\boldsymbol{\tau}) \frac{\mathcal{D} \boldsymbol{p}(\boldsymbol{\tau})}{(2 \pi \hbar)^{3}} e^{\frac{i}{\hbar} S} \\
& =\lim _{\boldsymbol{N} \rightarrow \infty} \prod_{j=1}^{N} \int_{-\infty}^{+\infty} d x_{j} d y_{j} d z_{j} \prod_{k=1}^{N+1} \int_{-\infty}^{+\infty} \frac{d^{3} p_{k}}{(2 \pi \hbar)^{3}} \exp \left\{\frac{i}{\hbar} S\right\} .
\end{aligned}
$$

Al ser reemplazados los componentes de $\boldsymbol{A}$ se obtiene el propagador:

$$
\begin{aligned}
\left\langle\boldsymbol{x}_{\boldsymbol{b}}, t_{b} \mid \boldsymbol{x}_{\boldsymbol{a}}, t_{a}\right\rangle= & \int_{-\infty}^{+\infty} \frac{d p_{z}}{2 \pi \hbar} \prod_{j=1}^{N} \int_{-\infty}^{+\infty} d x_{j} d y_{j} \prod_{k=1}^{N+1} \int_{-\infty}^{+\infty} \frac{d p_{x k} d p_{y k}}{(2 \pi \hbar)^{2}} \\
& \times \exp \left\{\frac{i}{\hbar}\left[p_{z}\left(z_{b}-z_{a}\right)-\left(t_{b}-t_{a}\right) \frac{p_{z}^{2}}{2 m}\right]\right\} \exp \left\{\frac{i}{\hbar} S_{R}\right\},
\end{aligned}
$$

donde $S_{R}$ es la acción reducida:

$$
\begin{gathered}
S_{R}=\sum_{j=1}^{N+1}\left\{p_{x j}\left(x_{j}-x_{j-1}\right)-\frac{\epsilon}{2 m} p_{x j}^{2}-\epsilon \frac{m \Omega^{2}}{2} x_{j}^{2}+p_{y j}\left(y_{j}-y_{j-1}\right)-\frac{\epsilon}{2 m} p_{y j}^{2}\right. \\
\left.-\epsilon \frac{m \Omega^{2}}{2} y_{j}^{2}+\epsilon \Omega\left(p_{y j} x_{j}-p_{x j} y_{j}\right)\right\} .
\end{gathered}
$$

La ecuación (5) representa la acción para un oscilador bidimensional con frecuencia $\Omega, y$ los dos últimos términos de la exponencial son las componentes $z$ del momento orbital angular. Sin embargo, sabemos que la trayectoria de la partícula en un campo magnético empleando la acción clásica es circular (para el caso $x y$ ) y para describir tal movimiento solo se requiere básicamente de la expresión para un oscilador armónico unidimensional. Es necesario replantear el procedimiento utilizado y buscar una alternativa en la transformación gauge que simplifique el procedimiento para emplear un oscilador unidimensional. Para hacer esto, descomponemos el vector potencial empleando la transformación $\boldsymbol{A}^{\prime}=\boldsymbol{A}(\boldsymbol{x})+\boldsymbol{\nabla} \alpha(\boldsymbol{x})$, donde $\alpha(\boldsymbol{x})$ es una función escalar arbitraria. En este caso el vector $\boldsymbol{A}(\boldsymbol{x})$ con componentes [6]

$$
A_{x}=0, A_{y}=B x, \quad A_{z}=0
$$

y la función $\alpha(\boldsymbol{x})=-(1 / 2) B x y$, permiten que el campo magnético sea descrito como $\boldsymbol{B}=\boldsymbol{\nabla} \times \boldsymbol{A}$. Este vector satisface la condición gauge de Coulomb $\nabla \boldsymbol{A}=0$ empleada para campos estáticos. 
CÁLCULO DEL PROPAGADOR CON EL VECTOR POTENCIAL $A(x)=(0, B x, 0)$

De acuerdo a la ecuación (3), el propagador

$$
\begin{aligned}
\left\langle\boldsymbol{x}_{\boldsymbol{b}}, t_{b} \mid \boldsymbol{x}_{\boldsymbol{a}}, t_{a}\right\rangle & =\int_{-\infty}^{+\infty} \mathcal{D} \boldsymbol{x}(\boldsymbol{\tau}) \frac{\mathcal{D} \boldsymbol{p}(\boldsymbol{\tau})}{(2 \pi \hbar)^{3}} e^{\frac{i}{\hbar} S} \\
& =\lim _{N \rightarrow \infty} \prod_{j=1}^{N} \int_{-\infty}^{+\infty} d x_{j} d y_{j} d z_{j} \prod_{k=1}^{N+1} \int_{-\infty}^{+\infty} \frac{d^{3} p_{k}}{(2 \pi \hbar)^{3}} \exp \left\{\frac{i}{\hbar} S\right\}
\end{aligned}
$$

utiliza la acción $S$ dada en (2), las cual se reemplaza por la acción discreta de tiempo, utilizando las componentes del vector potencial dados en (6),

$$
\begin{aligned}
S=\sum_{j=1}^{N+1}\left\{p_{x j}(\right. & \left.x_{j}-x_{j-1}\right)+p_{y j}\left(y_{j}-y_{j-1}\right)+p_{z j}\left(z_{j}-z_{j-1}\right) \\
- & \left.\frac{\epsilon}{2 m}\left[p_{x j}^{2}+p_{y j}^{2}+p_{z j}^{2}-\frac{2 e}{c} p_{y j} B_{x j}+\frac{e^{2}}{c^{2}} B^{2} x_{j}^{2}\right]\right\} .
\end{aligned}
$$

Se transforma esta expresión reordenando las variables $y_{j}, z_{j}$ de la siguiente manera:

$$
\begin{aligned}
\sum_{j=1}^{N+1} p_{y j}\left(y_{j}-\right. & \left.y_{j-1}\right)+p_{z j}\left(z_{j}-z_{j-1}\right)=p_{y(N+1)} y_{N+1}+p_{z(N+1)} z_{N+1}-p_{y 1} y_{0}-p_{z 1} z_{0} \\
& -\left(p_{y(N+1)}-p_{y N}\right) y_{N}-\left(p_{z(N+1)}-p_{z N}\right) z_{N}-\cdots-\left(p_{y 2}-p_{y 1}\right) y_{1} \\
& -\left(p_{z 2}-p_{z 1}\right) z_{1} .
\end{aligned}
$$

Utilizamos la transformada de Fourier de la función $\delta$ de Dirac para analizar esta relación. Podemos observar entonces, que para no obtener una solución trivial, las integrales de los $N$ momentos $p_{n}(n=1, \ldots, N)$ se evaluarán con la condición $p_{N}=p_{N-1}=\cdots=p_{1}$, por lo tanto la integración sobre las variables $y_{j}, z_{j}$ produce conduce a las funciones $\delta$ de Dirac:

$$
(2 \pi \hbar)^{2 N}\left[\delta\left(p_{y(N+1)}-p_{y N}\right) \delta\left(p_{z(N+1)}-p_{z N}\right) \cdots \delta\left(p_{y 2}-p_{y 1}\right) \delta\left(p_{z 2}-p_{z 1}\right)\right] .
$$

En consecuencia, la amplitud de transición puede ser representada de la forma:

$$
\begin{aligned}
\left\langle\boldsymbol{x}_{\boldsymbol{b}}, t_{b} \mid \boldsymbol{x}_{a}, t_{a}\right\rangle & =\int_{-\infty}^{+\infty} \frac{d p_{y} d p_{z}}{2 \pi \hbar} \prod_{j=1}^{N} \int_{-\infty}^{+\infty} d x_{j} \prod_{k=1}^{N+1} \int_{-\infty}^{+\infty} \frac{d p_{x k}}{(2 \pi \hbar)^{2}} \times \exp \left\{\frac { i } { \hbar } \left[p _ { y } \left(y_{b}\right.\right.\right. \\
& \left.\left.\left.-y_{a}\right)+p_{z}\left(z_{b}-z_{a}\right)-\left(t_{b}-t_{a}\right) \frac{p_{z}^{2}}{2 m}\right]\right\} \exp \left\{\frac{i}{\hbar} S_{R}\right\},
\end{aligned}
$$


40

en donde se ha obtenido la acción reducida para un oscilador armónico unidimensional con frecuencia $\omega=\frac{e B}{m c}$ (la frecuencia de ciclotrón),

$$
S_{R}=\sum_{j=1}^{N+1}\left\{p_{x j}\left(x_{j}-x_{j-1}\right)-\frac{\epsilon}{2 m} p_{x j}^{2}-\frac{\epsilon}{2 m}\left(p_{y}-\frac{e B}{c} x_{j}\right)^{2}\right\} .
$$

Integrando entonces la expresión (9) obtenemos

$$
\begin{aligned}
\left\langle\boldsymbol{x}_{\boldsymbol{b}}, t_{b} \mid \boldsymbol{x}_{a}, t_{a}\right\rangle & =\sqrt{\frac{m \omega}{2 \pi \hbar \operatorname{sen} \omega\left(t_{b}-t_{a}\right)}} \\
& \times \exp \left(\frac { i } { \hbar } \frac { m \omega } { 2 \operatorname { s e n } \omega ( t _ { b } - t _ { a } ) } \left\{\left[\left(x_{b}-x_{0}\right)^{2}+\left(x_{a}-x_{0}\right)^{2}\right] \cos \omega\left(t_{b}-t_{a}\right)\right.\right. \\
& \left.\left.-2\left(x_{b}-x_{0}\right)\left(x_{a}-x_{0}\right)\right\}\right) .
\end{aligned}
$$

Aquí aplicamos la solución para el oscilador armónico cuyo centro de oscilación $x_{0}=\frac{p_{y}}{m \omega}$ depende de $p_{y}$ [6], donde el radio $r=\frac{p}{q B}$ es el radio de Larmor.

Al evaluar la integral gaussiana en $p_{z}$ se obtiene la expresión

$$
\sqrt{\frac{m}{2 \pi i \hbar\left(t_{b}-t_{a}\right)}} \times \exp \left[\frac{i m\left(z_{b}-z_{a}\right)^{2}}{2 \hbar\left(t_{b}-t_{a}\right)}\right] .
$$

Por último evaluamos la integral en $p_{y}$ con $p_{y}=m \omega x_{0}$, obteniendo

$$
m \omega \int_{-\infty}^{+\infty} d x_{0} \exp \left\{\frac{i}{\hbar}\left[m \omega x_{0}\left(y_{b}-y_{a}\right)\right]\right\},
$$

y finalmente el propagador (9) toma la forma

$$
\begin{aligned}
\frac{i m \omega}{\hbar} x_{0}\left(y_{b}-y_{a}\right) & \\
+ & \frac{i}{\hbar} \frac{m \omega}{2 \operatorname{sen} \omega\left(t_{b}-t_{a}\right)}\left\{\left[\left(x_{b}-x_{0}\right)^{2}+\left(x_{a}-x_{0}\right)^{2}\right] \cos \omega\left(t_{b}-t_{a}\right)\right. \\
& \left.-2\left(x_{b}-x_{0}\right)\left(x_{a}-x_{0}\right)\right\} .
\end{aligned}
$$

Efectuando los productos, se obtiene la expresión para la exponencial 
41

$$
\begin{aligned}
\frac{\operatorname{im} \omega}{\hbar}\left\{x _ { 0 } \left(y_{b}-\right.\right. & \left.y_{a}\right)+\left(x_{b}^{2}+x_{a}^{2}\right) \frac{\cos \omega\left(t_{b}-t_{a}\right)}{2 \operatorname{sen} \omega\left(t_{b}-t_{a}\right)}-\left(x_{0} x_{b}+x_{0} x_{a}\right) \frac{\cos \omega\left(t_{b}-t_{a}\right)}{\operatorname{sen} \omega\left(t_{b}-t_{a}\right)} \\
& +x_{0}^{2} \frac{\cos \omega\left(t_{b}-t_{a}\right)}{2 \operatorname{sen} \omega\left(t_{b}-t_{a}\right)}-\frac{x_{b} x_{a}}{\operatorname{sen} \omega\left(t_{b}-t_{a}\right)}+\frac{x_{b} x_{0}}{\operatorname{sen} \omega\left(t_{b}-t_{a}\right)}+\frac{x_{a} x_{0}}{\operatorname{sen} \omega\left(t_{b}-t_{a}\right)} \\
& \left.-\frac{x_{0}^{2}}{\operatorname{sen} \omega\left(t_{b}-t_{a}\right)}\right\} .
\end{aligned}
$$

Simplificamos esta expresión y la llevamos a la forma clásica ya conocida [8]. Para esto, expresamos toda la suma en términos de la tangente del ángulo medio y completamos cuadrados en $x_{0}$, obteniendo la expresión

$$
\begin{aligned}
\frac{i m \omega}{2 \hbar}\left\{-\left(x_{b}^{2}+\right.\right. & \left.\left.x_{a}^{2}\right) \tan \left[\omega\left(t_{b}-t_{a}\right) / 2\right]+\frac{\left(x_{b}-x_{a}\right)^{2}}{\operatorname{sen} \omega\left(t_{b}-t_{a}\right)}\right\} \\
& -\frac{i m \omega}{\hbar} \tan \left[\omega\left(t_{b}-t_{a}\right) / 2\right]\left(x_{0}-\frac{x_{b}+x_{a}}{2}-\frac{y_{b}-y_{a}}{2 \tan \left[\omega\left(t_{b}-t_{a}\right) / 2\right]}\right)^{2} \\
& +\frac{i m \omega}{2 \hbar}\left[\frac{\left(x_{b}+x_{a}\right)^{2}}{2} \tan \left[\omega\left(t_{b}-t_{a}\right) / 2\right]+\frac{\left(y_{b}-y_{a}\right)^{2}}{2 \tan \left[\omega\left(t_{b}-t_{a}\right) / 2\right]}\right. \\
& \left.+\frac{i m \omega}{2 \hbar}\left(x_{b}+x_{a}\right)\left(y_{b}-y_{a}\right)\right]
\end{aligned}
$$

A partir de la integral gaussiana en $x_{0}$, se obtiene el factor:

$$
\frac{m \omega}{2 \pi \hbar} \sqrt{\frac{\pi \hbar}{i m \omega \tan \left[\omega\left(t_{b}-t_{a}\right) / 2\right]}}
$$

Finalmente se obtiene el propagador,

$$
\begin{aligned}
\left\langle\boldsymbol{x}_{\boldsymbol{b}}, t_{b} \mid \boldsymbol{x}_{a}, t_{a}\right\rangle & =\left(\frac{m}{2 \pi i \hbar\left(t_{b}-t_{a}\right)}\right)^{\frac{3}{2}} \frac{\omega\left(t_{b}-t_{a}\right) / 2}{\operatorname{sen}\left[\omega\left(t_{b}-t_{a}\right) / 2\right]} \\
& \times \exp \left\{\frac { i } { \hbar } \frac { m } { 2 } \left[\frac{i m\left(z_{b}-z_{a}\right)^{2}}{2 \hbar\left(t_{b}-t_{a}\right)}+\frac{\omega}{2} \cot \left[\omega\left(t_{b}-t_{a}\right) / 2\right]\left[\left(x_{b}-x_{a}\right)^{2}+\left(y_{b}-y_{a}\right)^{2}\right]\right.\right. \\
& \left.\left.+\omega\left(x_{a} y_{b}-x_{b} y_{a}\right)+\omega\left(x_{b} y_{b}-x_{a} y_{a}\right)\right]\right\}
\end{aligned}
$$

en donde el último término que aparece en la exponencial no está presente en la expresión clásica, ya que allí se emplea el vector potencial $\boldsymbol{A}^{\prime}=\frac{1}{2} \boldsymbol{B} \times \boldsymbol{r}$. y es denominado término de superficie [4]. La misma situación se puede observar en el caso de la acción 
42

para la integral de caminos dada en el espacio de configuraciones cuando la acción se expresa en términos del lagrangiano [7].

\section{CONCLUSIÓN}

La técnica de las integrales de camino en el espacio fase permitió la obtención de un resultado más preciso, con una componente de superficie, mostrando que existe una fuerte relación entre el hamiltoniano y la varianza gauge empleada para describir el campo magnético. La integral de camino para un oscilador armónico resulta matemáticamente más sencilla en su solución que con métodos de la mecánica clásica. Es importante recalcar, que a su vez la comprensión del comportamiento del oscilador armónico contribuye a problemas más complejos como el caso de una partícula cargada en un campo magnético y otros no expresados en el presente trabajo. La parte con mayor dificultad reside en conciliar el resultado obtenido con el resultado clásico donde el trabajo algebraico y trigonométrico puede ser bastante tedioso, pero en general la expresión para el propagador está también sustentada por integrales gaussianas que facilitan el resultado. Se ha demostrado además en el presente trabajo que al abordar el problema para la partícula en un espacio tridimensional, su solución se reduce prácticamente a la solución de un problema unidimensional. El rol del vector potencial en este proceso es crucial ya que a partir de él resultado se simplifica a una sola dimensión.

\section{Referencias bibliograficas}

1.- MacKenzie R. Path Integral Methods and Applications arXiv:quant-ph/0004090

2.- Müller-Kirsten H. Introduction to Quantum Mechanics Schrödinger Equation and Path Integral. World Scientific Publishing, 2006.

3.- Baaquie BE. Path Integrals and Hamiltonians. Cambridge University Press. United Kingdom, 2014.

4.- Kleinert H: Path Integrals in Quantum Mechanics, Statistics and Polymer Physics World Scientific, Singapore, 1990

5.-Chaichian M and A. Demichev A. Path Integrals in Physics, vol 1: Stochastic Processes and Quantum Mechanics. Institute of Physics Publishing. Londres, 2001

6.- Landau LD. Quantum Mechanic, Non-relativistic Theory. Pergamon Press. Great Britain, 1989

7.- Feynman RP and Hibbs AR. Quantum Mechanics and Path Integrals, Emended Edition. Dover Publications, New York, 2005

8.- Dittrich W and Reuter M. Classical and Quantum Dynamics. Springer, New York, 2001 